\title{
EPIDEMIC RISK AND INSURANCE COVERAGE
}

\author{
CLAUDE LEFÈVRE, ${ }^{* *}$ Université Libre de Bruxelles \\ PHILIPPE PICARD, ${ }^{* * *}$ Université de Lyon \\ MATTHIEU SIMON, ${ }^{* * * * *}$ Université Libre de Bruxelles
}

\begin{abstract}
In this paper we aim to apply simple actuarial methods to build an insurance plan protecting against an epidemic risk in a population. The studied model is an extended SIR epidemic in which the removal and infection rates may depend on the number of registered removals. The costs due to the epidemic are measured through the expected epidemic size and infectivity time. The premiums received during the epidemic outbreak are measured through the expected susceptibility time. Using martingale arguments, a method by recursion is developed to calculate the cost components and the corresponding premium levels in this extended epidemic model. Some numerical examples illustrate the effect of removals and the premium calculation in an insurance plan.
\end{abstract}

Keywords: SIR epidemic; removal-dependent rate; epidemic size; infectivity time; susceptibility time; insurance premium; martingale

2010 Mathematics Subject Classification: Primary 60J28; 91B30; 92D30

\section{Introduction}

Epidemics are a recurrent scourge in many countries that cause serious health problems and significant financial costs. There exists a growing need for insurance coverage and some companies are already offering to insure against the risk of epidemics and disease outbreaks.

Recently, traditional epidemic models have been proposed in different actuarial applications; see Chen and Cox (2009) and Feng and Garrido (2011). The present work is concerned with a Markovian epidemic model of the SIR (susceptible $\rightarrow$ infected $\rightarrow$ removed) type. Our purpose is to apply simple actuarial methods used in life insurance for a population that is exposed to such an epidemic outbreak.

An epidemic with removal-dependent rates. A variety of stochastic models have been proposed to investigate the spread of infectious diseases. See, e.g. Daley and Gani (1999) and Andersson and Britton (2000). A classical SIR epidemic model is the continuous-time Markov process named the general epidemic. In this model, each infective remains infectious during a period of time exponentially distributed of (constant) parameter $\mu$. While infected, it can contact any susceptible according to a Poisson process of (constant) rate $\beta$. At the end of the infectious period, it is immunized and removed with no further role in the epidemic process.

The epidemic model studied here is an extended general epidemic in which the removal and infection rates are allowed to depend on the number of registered removals. Let $S_{t}, I_{t}$,

\footnotetext{
Received 7 December 2015; revision received 11 May 2016.

* Postal address: Département de Mathématique, Université Libre de Bruxelles, Campus de la Plaine C.P. 210 , B-1050 Bruxelles, Belgium.

** Email address: clefevre@ulb.ac.be Also at the ISFA, Université de Lyon.

*** Postal address: ISFA, Université de Lyon, 50 Avenue Tony Garnier, F-69007 Lyon, France.

Email address: philippe.picard69@free.fr

**** Email address: matsimon@ulb.ac.be
} 
and $R_{t}$ be the numbers of suceptibles, infectives, and removed cases at time $t \geq 0$. Initially, $S_{0}=n, I_{0}=m$, and $R_{0}=0$, with $N=n+m$ as total size. The transition probabilities during $(t, t+\mathrm{d} t)$ are defined by

$$
\begin{gathered}
\mathbb{P}\left[S_{t+\mathrm{d} t}=s-1, I_{t+\mathrm{d} t}=i+1, R_{t+\mathrm{d} t}=r \mid S_{t}=s, I_{t}=i, R_{t}=r\right]=\beta_{r} s i \mathrm{~d} t+o(\mathrm{~d} t), \\
\mathbb{P}\left[S_{t+\mathrm{d} t}=s, I_{t+\mathrm{d} t}=i-1, R_{t+\mathrm{d} t}=r+1 \mid S_{t}=s, I_{t}=i, R_{t}=r\right]=\mu_{r} i \mathrm{~d} t+o(\mathrm{~d} t),
\end{gathered}
$$

all the infection rates $\beta_{r}$ and removal rates $\mu_{r}$ being positive.

Introducing rates $\beta_{r}$ and $\mu_{r}$ that depend upon the numbers of removed cases enables us to reflect different possible changes in response to the progress of the epidemic. This is especially true for sexually transmitted diseases. For instance, a number of individuals could react by reducing their risk of infection, giving a decreasing function for $\beta_{r}$. However, when more effective drugs are developed, treated patients could be indulging in more risky sexual behaviour, implying an inverse effect on the function $\beta_{r}$. In parallel, the worsening of the disease would then be slowed down, so that the function $\mu_{r}$ is decreasing. Nevertheless, the detection of infected cases is generally improving, hence again implying an inverse effect on $\mu_{r}$. So, the influence of the number of removals on the rates can be very diverse in practice.

An additional interest in considering a rate $\beta_{r}$ that is a function of $r$ is to cover at the same time the standard general epidemic and a well-known variant named the fatal epidemic (due to Gleissner (1988)). For the general epidemic, the removal of an infective corresponds to its immunization, so that the total population size remains constant over time; a usual assumption is then to write $\beta_{r} \equiv \beta=\alpha / N$, say, independently of $r$. For the fatal epidemic, the removal of an infective corresponds to its death, so that the surviving population decreases over time; in that case, the natural assumption is to set $\beta_{r}=\alpha /(N-r)$.

Epidemic models with removal-dependent rates have been little discussed so far. A noteworthy exception is O'Neill (1997) who investigated a fatal epidemic with a removal-dependent infection rate.

Organization of the work. Our motivation comes from current concerns about the coverage of an epidemic risk in large companies. On the one hand, the occurrence of an epidemic implies the reimbursement of medical expenses to the infected individuals during the period of their treatment, and possibly the payment of a lump sum to the removed individuals (in case of death or disability, for instance). On the other hand, the policyholders committed to paying premiums are all the susceptible individuals as long as they remain healthy. The level of premium is then obtained by applying the classical equivalence principle in life insurance, which requires that

$$
\mathbb{E}(\text { benefit outgo })=\mathbb{E}(\text { premium income }) .
$$

The time horizon taken into account is the whole duration of the epidemic, i.e. until the first instant $T$ when there are no more infectives in the population. Note that no force of interest has been incorporated in (1.1), which is an acceptable hypothesis when the outbreak does not last very long (as for mild diseases like influenza).

Sections 2 and 3 are concerned with the SIR model above in which the rates are removaldependent rates. In Section 2 we construct a family of martingales to obtain the joint distribution of the total epidemic size and infectivity time. This will allow us to calculate the mean benefit outgo in the left-hand side of (1.1). In Section 3, by again applying a martingale argument, we develop a recursive method to determine the expected susceptibility time. This will provide us with the mean premium income in the right-hand side of (1.1). It is worth mentioning that the susceptibility time has not been discussed so far in the epidemic literature. A more 
comprehensive study on this time will be the object of a future work. In Section 4 the analysis is continued for the general and fatal epidemics. Using generating functions, we derive an (almost) explicit solution to the recursion for the expected susceptibility time. In Section 5 we present three numerical examples for illustration: the general and fatal epidemics, a case with exponentially-dependent rates, and a model with a single change in the infection rate. We conclude in Section 6.

\section{Infectivity time}

A key component of the cost of the epidemic is the area under the trajectory of the infectives

$$
A_{t}=\int_{0}^{t} I_{u} \mathrm{~d} u, \quad t \geq 0,
$$

which represents the sum of all the personal units of infection until time $t$. This area allows us to measure the medical expenses necessary for providing care to the infected individuals. Another important cost component is given by the number of removed cases until $t$ when these individuals are dead or in disability, partial or permanent.

Our attention is focused on these two random variables evaluated at the end of the epidemic, i.e. $A_{T}$ and $R_{T}=N-S_{T}$. Much research has been devoted to the study of these statistics for standard SIR models. For the general epidemic, we refer the reader to, e.g. Gani and Jerwood (1972), Ball (1986), and Picard and Lefèvre (1990); for the fatal epidemic, see Picard and Lefèvre (1993) and Ball and O’Neill (1993).

We are going to determine the joint distribution of $\left(S_{T}, A_{T}\right)$ for the extended epidemic with removal-dependent rates presented before. To this end, our first step is to find a real function $a(s, i ; \theta)$, where $0 \leq s \leq n, 0 \leq i \leq n+m$ are integers and $\theta \geq 0$, such that the process

$$
M_{t} \equiv a\left(S_{t}, I_{t} ; \theta\right) \mathrm{e}^{-\theta A_{t}}, \quad t \geq 0,
$$

forms a martingale with respect to the filtration $\mathcal{F}_{t}=\sigma\left\{\left(S_{u}, I_{u}\right), 0 \leq u \leq t\right\}$. Let us recall that $\left(S_{t}=s, I_{t}=i\right)$ yields $R_{t}=r$ with $r=N-s-i$.

Lemma 2.1. It holds that $\left\{M_{t}, t \geq 0\right\}$ is a martingale if the $a(s, i ; \theta)$ satisfy the conditions

$$
\left(\theta+\beta_{r} s+\mu_{r}\right) a(s, i ; \theta)=\beta_{r} s a(s-1, i+1 ; \theta)+\mu_{r} a(s, i-1 ; \theta), \quad i \geq 1,
$$

where $a(-1, i+1 ; \theta)=0$, say. These relations define $a(s, i ; \theta)$ recursively once the $a(s, 0 ; \theta)$ are fixed.

Proof. Following the method of Picard (1980), we introduce

$$
m_{t}=\mathbb{E}\left(M_{t} \mid \mathcal{F}_{t_{0}}\right), \quad t \geq t_{0}
$$

for any fixed $t_{0} \geq 0$. By examining the time interval $(t, t+\mathrm{d} t)$, we have

$$
\begin{aligned}
m_{t+\mathrm{d} t}= & \mathbb{E}\left\{\left[\beta_{R_{t}} S_{t} I_{t} a\left(S_{t}-1, I_{t}+1 ; \theta\right) \mathrm{d} t+\mu_{R_{t}} I_{t} a\left(S_{t}, I_{t}-1 ; \theta\right) \mathrm{d} t\right] \mathrm{e}^{-\theta A_{t}}\right. \\
& \left.+a\left(S_{t}, I_{t} ; \theta\right)\left[1-\beta_{R_{t}} S_{t} I_{t} \mathrm{~d} t-\mu_{R_{t}} I_{t} \mathrm{~d} t\right]\left[1-\theta I_{t} \mathrm{~d} t\right] \mathrm{e}^{-\theta A_{t}}+o(\mathrm{~d} t) \mid \mathcal{F}_{t_{0}}\right\} .
\end{aligned}
$$

Letting $\mathrm{d} t \rightarrow 0$, we easily see that the right derivative $m_{t}^{\prime}$ exists and is given by

$$
\begin{aligned}
m_{t}^{\prime}= & \mathbb{E}\left\{\left[\beta_{R_{t}} S_{t} I_{t}\left(a\left(S_{t}-1, I_{t}+1 ; \theta\right)-a\left(S_{t}, I_{t} ; \theta\right)\right)\right.\right. \\
& \left.\left.+\mu_{R_{t}} I_{t}\left(a\left(S_{t}, I_{t}-1 ; \theta\right)-a\left(S_{t}, I_{t} ; \theta\right)\right)-\theta I_{t} a\left(S_{t}, I_{t} ; \theta\right)\right] \mathrm{e}^{-\theta A_{t}} \mid \mathcal{F}_{t_{0}}\right\} .
\end{aligned}
$$


If $a(s, i ; \theta)$ is chosen such that $m_{t}^{\prime}=0$, then $m_{t}=m_{t_{0}}$ for all $t \geq t_{0}$ so that $\left\{M_{t}, t \geq 0\right\}$ is a martingale. For that, it suffices to require that for all $s, i$,

$$
\beta_{r} \operatorname{si}[a(s-1, i+1 ; \theta)-a(s, i ; \theta)]+\mu_{r} i[a(s, i-1 ; \theta)-a(s, i ; \theta)]=\theta i a(s, i ; \theta),
$$

where $a(-1, i+1 ; \theta)=a(s,-1 ; \theta)=0$, say. This condition is obviously satisfied for $i=0$. For $i \geq 1$, it can be written as relation (2.2) after dividing by $i$. For the recursion, it suffices to begin with $s=0$ and $i=1,2, \ldots, n+m$ starting with a given value for $a(0,0 ; \theta)$, then pass to $s=1$ and $i=1,2, \ldots, n+m-1$ from a given value $a(1,0 ; \theta)$, and continue in the same way by increasing $s$ until $n$.

Let us now look for an explicit solution to (2.2), if it exists. In fact, we will find $n+1$ solutions which provide us with a family of $n+1$ martingales.

Proposition 2.1. For any integer $k \in[0, n]$, the associated process

$$
M_{t}(k) \equiv\left(\begin{array}{l}
S_{t} \\
k
\end{array}\right) \prod_{j=1}^{R_{t}}\left(1+\frac{\theta+k \beta_{j-1}}{\mu_{j-1}}\right) \mathrm{e}^{-\theta A_{t}}, \quad t \geq 0,
$$

constitutes a martingale.

Proof. Fix any integer $k$ in $[0, n]$. We want to build an associated function $a(\cdot)$, denoted $a_{k}(s, i ; \theta)$, of the form

$$
a_{k}(s, i ; \theta) \equiv\left(\begin{array}{l}
s \\
k
\end{array}\right) \prod_{j=0}^{N-s-i} g_{j}(k, \theta)
$$

where $g_{0}(\cdot)=1$ and $g_{j}(k, \theta)$ are positive functions to be chosen. As before, we write $r=$ $N-s-i$, with $0 \leq r \leq N-1$. Substituting (2.4) in (2.2) then yields

$$
\left(\theta+\beta_{r} s+\mu_{r}\right)\left(\begin{array}{l}
s \\
k
\end{array}\right) \prod_{j=0}^{r} g_{j}(k, \theta)=\beta_{r} s\left(\begin{array}{c}
s-1 \\
k
\end{array}\right) \prod_{j=0}^{r} g_{j}(k, \theta)+\mu_{r}\left(\begin{array}{l}
s \\
k
\end{array}\right) \prod_{j=0}^{r+1} g_{j}(k, \theta) .
$$

After division by $\left(\begin{array}{l}s \\ k\end{array}\right) \prod_{j=0}^{r} g_{j}(k, \theta),(2.5)$ can be expressed as

$$
\theta+\beta_{r} s+\mu_{r}=\beta_{r}(s-k)+\mu_{r} g_{r+1}(k, \theta),
$$

which reduces to

$$
\theta+\beta_{r} k+\mu_{r}=\mu_{r} g_{r+1}(k, \theta)
$$

so that

$$
g_{r+1}(k, \theta)=\frac{\theta+\beta_{r} k+\mu_{r}}{\mu_{r}} .
$$

Thus, the function $a_{k}(\cdot)$ in (2.4) is completely defined as

$$
a_{k}(s, i ; \theta)=\left(\begin{array}{l}
s \\
k
\end{array}\right) \prod_{j=1}^{N-s-i}\left(1+\frac{\theta+k \beta_{j-1}}{\mu_{j-1}}\right) .
$$

Inserting (2.6) in (2.1) then gives the martingale (2.3).

We are in a position to determine the joint distribution of the statistics $\left(S_{T}, A_{T}\right)$ at the end $T$ of the epidemic. 
Proposition 2.2. For $k=0, \ldots, n$,

$$
\mathbb{E}\left[\left(\begin{array}{c}
S_{T} \\
k
\end{array}\right) \prod_{j=1}^{N-S_{T}}\left(1+\frac{\theta+k \beta_{j-1}}{\mu_{j-1}}\right) \mathrm{e}^{-\theta A_{T}}\right]=\left(\begin{array}{l}
n \\
k
\end{array}\right) .
$$

When $\theta=0$ in (2.7), we have

$$
\mathbb{E}\left[\left(\begin{array}{c}
S_{T} \\
k
\end{array}\right) \prod_{j=1}^{N-S_{T}}\left(1+\frac{k \beta_{j-1}}{\mu_{j-1}}\right)\right]=\left(\begin{array}{l}
n \\
k
\end{array}\right) .
$$

Considering successively $k=n, \ldots, 1,(2.8)$ forms a triangular system of $n$ linear equations in the state probabilities $\mathbb{P}\left(S_{T}=s\right), 1 \leq s \leq n$, giving then also $\mathbb{P}\left(S_{T}=0\right)$.

When $k=0$ in (2.7),

$$
\mathbb{E}\left[\prod_{j=1}^{N-S_{T}}\left(1+\frac{\theta}{\mu_{j-1}}\right) \mathrm{e}^{-\theta A_{T}}\right]=1
$$

yielding, e.g.

$$
\mathbb{E}\left(A_{T}\right)=\mathbb{E}\left(\sum_{j=1}^{N-S_{T}} \frac{1}{\mu_{j-1}}\right) .
$$

Proof. Applying the optional stopping theorem, we obtain, from (2.3),

$$
\mathbb{E}\left[M_{T}(k)\right]=M_{0}(k),
$$

which yields (2.7) as $I_{T}=0$. Equation (2.8) directly follows. Differentiating (2.9) with respect to $\theta$ and putting $\theta=0$ gives (2.10).

Equation (2.10) for $\mathbb{E}\left(A_{T}\right)$ is easy to use, once the distribution of $S_{T}$ has been evaluated from (2.8). Note that if the removal rates $\mu_{r} \equiv \mu$ are independent of $r$, then

$$
\mathbb{E}\left(A_{T}\right)=\frac{N-\mathbb{E}\left(S_{T}\right)}{\mu} .
$$

In particular, (2.11) holds for the general and fatal epidemics. The expectations $\mathbb{E}\left(A_{T}\right)$ and $N-\mathbb{E}\left(S_{T}\right)$ are two cost measures useful in insurance (see Section 5).

\section{Susceptibility time}

The revenue in the epidemic comes essentially from the susceptibles still present. The area under the trajectory of the susceptibles

$$
B_{t}=\int_{0}^{t} S_{u} \mathrm{~d} u, \quad t \geq 0,
$$

represents the sum of all the personal units of susceptibility until time $t$. It provides us with a measure of the payments received from the insured persons.

To the best of the authors' knowledge, this area has not been examined in the epidemic literature. Our focus is, as before, on the end $T$ of the epidemic. Motivated by the actuarial 
applications, we will consider only the expected susceptibility time $\mathbb{E}\left(B_{T}\right)$. The joint distribution of $\left(S_{T}, B_{T}\right)$, however, can be determined using the framework developed here. This topic, which is rather technical, will be the subject of a future paper.

To begin with, we observe that

$$
B_{T}=\int_{0}^{T} S_{u} \mathrm{~d} u=\int_{0}^{T} S_{u} \mathbf{1}_{\left\{I_{u}>0\right\}} \mathrm{d} u,
$$

i.e. substituting $S_{u} \mathbf{1}_{\left\{I_{u}>0\right\}}$ for $S_{u}$ has no impact on the integral when it is taken until the end of the epidemic. So, instead of $B_{t}$, we choose to work with the integral

$$
\begin{aligned}
\int_{0}^{t} S_{u} \mathbf{1}_{\left\{I_{u}>0\right\}} \mathrm{d} u & =\int_{0}^{t} S_{u} \mathbf{1}_{\left\{S_{u} \geq 0, I_{u}>0\right\}} \mathrm{d} u \\
& =\sum_{0 \leq k \leq n, 1 \leq l \leq N-k} k \int_{0}^{t} \mathbf{1}_{\left\{S_{u}=k, I_{u}=l\right\}} \mathrm{d} u, \quad t \geq 0 .
\end{aligned}
$$

Let us write

$$
B_{t}(k, l) \equiv \int_{0}^{t} \mathbf{1}_{\left\{S_{u}=k, I_{u}=l\right\}} \mathrm{d} u, \quad t \geq 0
$$

for any fixed $0 \leq k \leq n, 1 \leq l \leq N-k$. At time $T, B_{T}(k, l)$ measures the time spent by the epidemic process in state $(k, l)$ if this state has been reached. Therefore, $B_{T}(k, l)$, if positive, has an exponential distribution with parameter $\beta_{N-k-l} k l+\mu_{N-k-l} l$. The probability $\chi_{k, l}=\mathbb{P}\left[B_{T}(k, l)>0\right]$ is of particular interest and will be provided by (3.12) below. As a consequence, we will then be able to calculate $\mathbb{E}\left[B_{T}(k, l)\right]$, and thus $\mathbb{E}\left(B_{T}\right)$ after insertion in (3.1) and (3.2).

To obtain the distribution of $B_{T}(k, l)$, we again follow a martingale approach. Specifically, we first find a martingale of a form similar to (2.1), i.e.

$$
M_{t} \equiv a\left(S_{t}, I_{t} ; \theta\right) \mathrm{e}^{-\theta B_{t}(k, l)}, \quad t \geq 0,
$$

with $\theta \geq 0$. By arguing as for Lemma 2.1, we obtain the following result.

Lemma 3.1. It holds that $\left\{M_{t}, t \geq 0\right\}$ is a martingale if the $a(s, i ; \theta)$ satisfy the conditions

$$
\left(\theta \delta_{s, k} \delta_{i, l}+\beta_{r} s i+\mu_{r} i\right) a(s, i ; \theta)=\beta_{r} \operatorname{sia}(s-1, i+1 ; \theta)+\mu_{r} i a(s, i-1 ; \theta), \quad i \geq 1,
$$

where $a(-1, i+1 ; \theta)=0$, say. These relations define $a(s, i ; \theta)$ recursively once the $a(s, 0 ; \theta)$ are fixed.

Note that as $l \geq 1$, (3.4) holds too for $i=0$ with $a(s,-1 ; \theta)=0$, say. Moreover, we have $a(s, i ; \theta) \equiv a_{k, l}(s, i ; \theta)$ as it depends on the value of $(k, l)$. Let us examine (3.4) in more detail.

Lemma 3.2. (i) For $s=0$,

$$
a(0, i ; \theta)= \begin{cases}a(0,0 ; \theta) & \text { if } k \geq 1 \text { or } k=0, i<l, \\ \frac{a(0,0 ; \theta) \mu_{N-l} l}{\theta+\mu_{N-l} l} & \text { if } k=0, i \geq l .\end{cases}
$$

(ii) If the $a(s, 0 ; \theta)$ are independent of $\theta$, this property holds too for the $a(s, i ; \theta)$ when $s<k$ or $s+i<k+l$. 
(iii) When $a(s, 0 ; \theta)=1$ for all $s, a(s, i ; \theta)$ can be expressed as

$$
a(s, i ; \theta)= \begin{cases}1 & \text { if } s+i<k+l \text { or } s<k, \\ f(s, i)+\frac{g(s, i)}{\theta+\beta_{N-k-l} k l+\mu_{N-k-l} l} & \text { if } s+i \geq k+l \text { and } s \geq k,\end{cases}
$$

for appropriate real functions $f$ and $g$.

In particular, if $s=k$ and $i=l$,

$$
a(k, l ; \theta)=\beta_{N-k-l} k l+\frac{\mu_{N-k-l} l}{\theta+\beta_{N-k-l} k l+\mu_{N-k-l} l},
$$

while if $\theta=0$, then $a(s, i ; 0)=1$ in all cases.

Proof. When $s=0,(3.4)$ reduces to

$$
\left(\theta \delta_{0, k} \delta_{i, l}+\mu_{r} i\right) a(0, i ; \theta)=\mu_{r} i a(0, i-1 ; \theta), \quad i \geq 1 .
$$

Thus, for $k \geq 1$, the $a(0, i ; \theta)$ are all equal to $a(0,0 ; \theta)$. For $k=0$,

$$
a(0, i ; \theta)=\frac{a(0, i-1 ; \theta) \mu_{r} i}{\theta \delta_{i, l}+\mu_{r} i}
$$

and considering $i<l$ and $i \geq l$ leads to (3.5). Now, each $a(s, i ; \theta)$ is a linear function of $a(s-1, i+1 ; \theta)$ and $a(s, i-1 ; \theta)$ with coefficients that are independent of $\theta$ except when $s=k$ and $i=l$. This yields the assertion of (ii). Finally, from the recursion (3.4) and (ii), we see that when the $a(s, 0 ; \theta)$ are equal to 1 , a general expression for $a(s, i ; \theta)$ is indeed given by (3.6). When $\theta=0,(3.4)$ shows that $k$ and $l$ have no real role, so that taking them arbitrarily large yields $a(s, i ; 0)=1$ by the first identity of (3.6).

In the sequel, we choose $a(s, 0 ; \theta)=1$ for all $s$, as in (iii) above. For clarity, the dependence on $(k, l)$ is now made explicit in the notation. Let us define

$$
b_{k, l}(s, i)=\left[\frac{\mathrm{d}}{\mathrm{d} \theta} a_{k, l}(s, i ; \theta)\right]_{\theta=0} .
$$

We show below how $\mathbb{E}\left(B_{T}\right)$ can be computed using the $b_{k, l}(n, m)$ for all $k, l$.

Proposition 3.1. We have

$$
\mathbb{E}\left[\mathrm{e}^{-\theta B_{T}(k, l)}\right]=a_{k, l}(n, m ; ; \theta),
$$

yielding, in particular,

$$
\mathbb{E}\left[B_{T}(k, l)\right]=-b_{k, l}(n, m)
$$

As a consequence,

$$
\mathbb{E}\left(B_{T}\right)=-\sum_{0 \leq k \leq n} \sum_{1 \leq l \leq N-k} k b_{k, l}(n, m) .
$$

Proof. By the optional stopping theorem,

$$
\mathbb{E}\left[M_{T}(k, l)\right]=a_{k, l}(n, m ; \theta),
$$

which gives (3.8) since $a_{k, l}\left(S_{T}, 0 ; \theta\right)=1$ by assumption. Differentiating (3.8) with respect to $\theta$ yields (3.9), in the notation of (3.7). As for (3.10), it is directly obtained from (3.1), (3.2), and (3.9).

Thanks to (3.8), we can also deduce the complete distribution of $B_{T}(k, l)$. 
Proposition 3.2. We have

$$
\mathbb{E}\left[\mathrm{e}^{-\theta B_{T}(k, l)}\right]=1-\chi_{k, l}+\chi_{k, l} \frac{\beta_{N-k-l} k l+\mu_{N-k-l} l}{\theta+\beta_{N-k-l} k l+\mu_{N-k-l} l},
$$

where $\chi_{k, l} \in(0,1)$ is defined by

$$
\chi_{k, l}=-b_{k, l}(n, m)\left(\beta_{N-k-l} k l+\mu_{N-k-l} l\right) .
$$

As announced after (3.3), $B_{T}(k, l)$ has the distributional representation

$$
B_{T}(k, l) \stackrel{\mathrm{D}}{=} Y \mathbf{1}_{\{W>0\}},
$$

where the random variable $Y$ has an exponential distribution with parameter $\beta_{N-k-l} k l+$ $\mu_{N-k-l} l$, and the random variable $W$ is independent of $Y$ and is nonnegative with $\mathbb{P}(W>$ $0)=\chi_{k, l}$.

Proof. Since $a_{k, l}(s, i ; 0)=1,(3.6)$ with $\theta=0$ implies that

$$
1=f_{k, l}(s, i)+\frac{g_{k, l}(s, i)}{\beta_{N-k-l} k l+\mu_{N-k-l} l} .
$$

So, (3.6) for $\theta \geq 0$ can be written as

$$
a_{k, l}(s, i ; \theta)=1-\frac{g_{k, l}(s, i)}{\beta_{N-k-l} k l+\mu_{N-k-l} l}+\frac{g_{k, l}(s, i)}{\theta+\beta_{N-k-l} k l+\mu_{N-k-l} l} .
$$

Moreover, by definition, we obtain

$$
b_{k, l}(s, i)=-\frac{g_{k, l}(s, i)}{\left(\beta_{N-k-l} k l+\mu_{N-k-l} l\right)^{2}} .
$$

Thus, expressing (3.13) in terms of $b_{k, l}(s, i)$, we have

$$
a_{k, l}(s, i ; \theta)=1-\frac{b_{k, l}(s, i)\left(\beta_{N-k-l} k l+\mu_{N-k-l} l\right)^{2}}{\theta+\beta_{N-k-l} k l+\mu_{N-k-l} l}+b_{k, l}(s, i)\left(\beta_{N-k-l} k l+\mu_{N-k-l} l\right) .
$$

From (3.8), we choose $s=n$ and $i=m$ in (3.14) and then obtain

$\mathbb{E}\left[\mathrm{e}^{-\theta B_{T}(k, l)}\right]=1+b_{k, l}(n, m)\left(\beta_{N-k-l} k l+\mu_{N-k-l} l\right)-\frac{b_{k, l}(n, m)\left(\beta_{N-k-l} k l+\mu_{N-k-l} l\right)^{2}}{\theta+\beta_{N-k-l} k l+\mu_{N-k-l} l}$,

which can be rewritten as (3.11) using the notation of (3.12). Finally, taking $\theta=\infty$ in (3.11), we see that

$$
\mathbb{P}\left[B_{T}(k, l)=0\right]=1-\chi_{k, l},
$$

so that $0<\chi_{k, l}<1$. The distributional representation for $B_{T}(k, l)$ directly follows.

To apply (3.10) and (3.11), the terms $b_{k, l}(n, m)$ need to be determined. This can be achieved recursively as for $a_{k, l}(n, m ; \theta)$. Specifically, (3.4) and Lemma 3.2 easily yield the following result. 
Proposition 3.3. The $b_{k, l}(s, i)$ satisfy the recursion

$$
\left(\beta_{r} s i+\mu_{r} i\right) b_{k, l}(s, i)=\beta_{r} s i b_{k, l}(s-1, i+1)+\mu_{r} i b_{k, l}(s, i-1)-\delta_{s, k} \delta_{i, l}, \quad i \geq 1,
$$

where $b_{k, l}(-1, i+1)=0$, say, and starting with $b_{k, l}(s, 0)=0$ for all $s$.

In particular, when $s=0$,

$$
b_{k, l}(0, i)= \begin{cases}0 & \text { if } k \geq 1 \text { or } k=0, i<l, \\ -\frac{1}{\mu_{N-l} l} & \text { if } k=0, i \geq l,\end{cases}
$$

and when $s>0$,

$$
b_{k, l}(s, i)= \begin{cases}0 & \text { if } s+i<k+l \text { or } s<k, \\ -\frac{1}{\beta_{N-k-l} k l+\mu_{N-k-l} l} & \text { if } s=k, i=l .\end{cases}
$$

Moreover, all the $b_{k, l}(s, i)$ are negative or null.

\section{General and fatal epidemics}

Let us continue the previous analysis for the two most classical SIR epidemic models. More precisely, we will derive an (almost) explicit solution to the recursion (3.15) giving the $b_{k, l}(s, i)$. The structure of the solution is simple and similar for both models.

General epidemic model. The rates are $\beta_{r}=\beta$ and $\mu_{r}=\mu$, i.e. independent of $r$. Then (3.15) can be written as

$$
(\beta s i+\mu i) b_{k, l}(s, i)=\beta s i b_{k, l}(s-1, i+1)+\mu i b_{k, l}(s, i-1)-\delta_{s, k} \delta_{i, l}, \quad i \geq 1,
$$

with (3.16) and (3.17) simplified accordingly. Let us define, for $u, v \geq 0$,

$$
\pi_{u, v}(s, i)=\left(\begin{array}{l}
s \\
u
\end{array}\right) \sum_{j=u}^{s}(-1)^{j-u+1}\left(\begin{array}{l}
s-u \\
s-j
\end{array}\right) \frac{\mu^{s+i-u-v}}{(\mu+\beta j)^{s+i-u-v+1}} .
$$

Proposition 4.1. Let $s \geq k$ and $s+i \geq k+l$. For the general epidemic,

$$
b_{k, l}(s, i)=\frac{1}{l} \pi_{k, l}(s, i)+\beta \sum_{j=k+l}^{s} b_{k, l}(j-1,1) j \pi_{j, 0}(s, i), \quad i \geq 1 .
$$

If $k+l>s$, (4.3) reduces to $b_{k, l}(s, i)=(1 / l) \pi_{k, l}(s, i)$. If $k+l \leq s$, the unknown $b_{k, l}(j-1,1)$ in (4.3) are first computed through the recursion

$$
0=\frac{1}{l} \pi_{k, l}(\sigma, 0)+\beta \sum_{j=k+l}^{\sigma} b_{k, l}(j-1,1) j \pi_{j, 0}(\sigma, 0) \text { for } \sigma=k+l, \ldots, s .
$$

Proof. We may consider a sequence of $b_{k, l}(s, i)$ defined by (4.1) for all $i \geq 1$, and not only for $i \leq n+m-s$, still with $s \leq n$. These $b_{k, l}(s, i)$ are negative or null and we see, by recurrence from (4.1), that they remain bounded. So, it makes sense to define the generating function

$$
D_{k, l}(s)=\sum_{i=1}^{\infty} b_{k, l}(s, i) x^{i}, \quad 0<x<1 .
$$


Let us multiply (4.1) by $x^{i} / i$ and then sum over $i \geq 1$. Noting that

$$
\begin{gathered}
\sum_{i=1}^{\infty} b_{k, l}(s, i-1) x^{i}=x D_{k, l}(s)+x b_{k, l}(s, 0)=x D_{k, l}(s), \\
\sum_{i=1}^{\infty} b_{k, l}(s-1, i+1) x^{i}=\frac{1}{x} D_{k, l}(s-1)-b_{k, l}(s-1,1), \quad s \geq 1,
\end{gathered}
$$

we obtain, for $D_{k, l}(s)$, the recurrence formula

$$
(\beta s+\mu-\mu x) D_{k, l}(s)=\beta s \frac{1}{x} D_{k, l}(s-1)-\beta s b_{k, l}(s-1,1)-\frac{\delta_{s, k} x^{l}}{l} .
$$

Recalling that by (3.17), $b_{k, l}(s, i)=0$, and, thus, $D_{k, l}(s)=0$, when $s<k$. From (4.6), we easily deduce that, for $s \geq k$,

$$
\begin{aligned}
D_{k, l}(s)= & -\frac{x^{k+l-s} \beta^{s-k}}{l} \frac{s !}{k !} \prod_{j=k}^{s} \frac{1}{\beta j+\mu-\mu x} \\
& -\sum_{j=k+1}^{s} x^{j-s} b_{k, l}(j-1,1) \beta^{s-j+1} \frac{s !}{(j-1) !} \prod_{t=j}^{s} \frac{1}{\beta t+\mu-\mu x}, \quad s \geq k .
\end{aligned}
$$

By (3.15), $b_{k, l}(j-1,1)=0$ when $j<k+l$, so that the sum in (4.7) is in fact over $j \geq k+l$.

Now, the two products in (4.7) are rational fractions with distinct poles and a null integer part. They can be decomposed in simple elements and then expanded as a power series in $x$. For the first product, and similarly for the second, we have

$$
\prod_{j=k}^{s} \frac{1}{\beta j+\mu-\mu x}=\left(\frac{-1}{\mu}\right)^{s-k} \sum_{j=k}^{s} \frac{m_{j}(k, s)}{\beta j+\mu-\mu x}=\left(\frac{-1}{\mu}\right)^{s-k} \sum_{u=0}^{\infty} x^{u} \sum_{j=k}^{s} \frac{m_{j}(k, s) \mu^{u}}{(\beta j+\mu)^{u+1}},
$$

where the $m_{j}(k, s)$ are constants given by

$$
m_{j}(k, s)=\left[\prod_{u=k ; u \neq j}^{s} \frac{\beta}{\mu}(j-u)\right]^{-1}=\left(\frac{\mu}{\beta}\right)^{s-k}(-1)^{s-j} \frac{k !}{s !}\left(\begin{array}{l}
s \\
k
\end{array}\right)\left(\begin{array}{l}
s-k \\
s-j
\end{array}\right) .
$$

By inserting (4.8) and (4.9) in (4.7), we can rewrite $D_{k, l}(s)$ under the form

$$
D_{k, l}(s)=\frac{1}{l} \sum_{u=0}^{\infty} x^{u+k+l-s} c_{u}(k, s)+\sum_{j=k+l}^{s} b_{k, l}(j-1,1) \sum_{u=0}^{\infty} x^{u+j-s} d_{u}(j, s),
$$

the coefficients $c_{j}(k, s, u)$ and $d_{j}(k, s, u)$ being provided by

$$
\begin{gathered}
c_{u}(k, s)=-\left(\frac{-\beta}{\mu}\right)^{s-k} \frac{s !}{k !} \sum_{j=k}^{s} \frac{m_{j}(k, s) \mu^{u}}{(\beta j+\mu)^{u+1}}=\left(\begin{array}{l}
s \\
k
\end{array}\right) \sum_{j=k}^{s}(-1)^{j-k+1}\left(\begin{array}{l}
s-k \\
s-j
\end{array}\right) \frac{\mu^{u}}{(\beta j+\mu)^{u+1}}, \\
d_{u}(j, s)=-\beta\left(\frac{-\beta}{\mu}\right)^{s-j} \frac{s !}{(j-1) !} \sum_{t=j}^{s} \frac{m_{t}(j, s) \mu^{u}}{(\beta t+\mu)^{u+1}}=\beta j c_{u}(j, s) .
\end{gathered}
$$


Finally, to obtain the $b_{k, l}(s, i)$, the coefficients of $x^{i}, i \geq 0$, in the power series (4.5) and (4.10) are identified. For $i \geq 1$, we have

$$
b_{k, l}(s, i)=\frac{1}{l} c_{s+i-k-l}(k, s)+\beta \sum_{j=k+l}^{s} b_{k, l}(j-1,1) j c_{s+i-j}(j, s),
$$

which becomes (4.3) using the notation of (4.2). If $k+l>s$, the sum in (4.3) disappears. Otherwise, the $b_{k, l}(j-1,1)$ must be first evaluated. This is done by taking $i=0$ in $(4.3)$ with $b_{k, l}(s, 0)=0$, which yields the recursion (4.4).

Fatal epidemic model. The rates are $\beta_{r}=\alpha /(N-r)=\alpha /(s+i)$ and $\mu_{r}=\mu$. Then, (3.15) can be written as

$$
\left(\frac{\alpha s i}{s+i}+\mu i\right) b_{k, l}(s, i)=\frac{\alpha s i}{s+i} b_{k, l}(s-1, i+1)+\mu i b_{k, l}(s, i-1)-\delta_{s, k} \delta_{i, l}, \quad i \geq 1,
$$

with (3.16) and (3.17) adapted accordingly. Let $I\left(n_{1}, n_{2}, n_{3}\right)=\int_{0}^{1} v^{n_{1}}(1-v)^{n_{2}}\left(1-v^{\alpha / \mu}\right)^{n_{3}} \mathrm{~d} v$ be the hypergeometric integral (when it exists), and define, for $u, v \geq 0$,

$$
\phi_{u, v}(s, i)=-\frac{u+v}{\mu}\left(\begin{array}{l}
s \\
u
\end{array}\right)\left(\begin{array}{l}
s+i \\
u+v
\end{array}\right) I\left[u\left(1+\frac{\alpha}{\mu}\right)+v-1, s+i-u-v, s-u\right] .
$$

Proposition 4.2. Let $s \geq k$ and $s+i \geq k+l$. For the fatal epidemic,

$$
b_{k, l}(s, i)=\frac{1}{l} \phi_{k, l}(s, i)+\alpha \sum_{j=k+l}^{s} b_{k, l}(j-1,1) \phi_{j, 0}(s, i), \quad i \geq 1 .
$$

If $k+l>s$, (4.13) reduces to $b_{k, l}(s, i)=(1 / l) \phi_{k, l}(s, i)$. If $k+l \leq s$, the unknown $b_{k, l}(j-1,1)$ in (4.13) are first computed through the recursion

$$
0=\frac{1}{l} \phi_{k, l}(\sigma, 0)+\alpha \sum_{j=k+l}^{\sigma} b_{k, l}(j-1,1) \phi_{j, 0}(\sigma, 0) \quad \text { for } \sigma=k+l, \ldots, s .
$$

Proof. Let us consider a sequence of $b_{k, l}(s, i)$ defined by (4.11) for all $s \geq 0, i \geq 1$, with the border condition $b_{k, l}(s, 0)=0$ (also when $s>n$ ). As these extended $b_{k, l}(s, i)$ are bounded, we may introduce the two generating functions

$$
\begin{array}{cl}
G_{k, l}(x, y)=\sum_{s=0}^{\infty} \sum_{i=1}^{\infty} b_{k, l}(s, i) \frac{x^{s}}{s !} \frac{y^{s+i}}{s+i}, & x \text { real, } 0 \leq y<1, \\
M_{k, l}(z)=\sum_{s=1}^{\infty} b_{k, l}(s-1,1) \frac{z^{s}}{s !}, & 0 \leq z<1 .
\end{array}
$$

To determine $G_{k, l}(x, y)$, we multiply (4.11) by $\left(x^{s} / s !\right)\left(y^{s+i} / i\right)$ and then sum over $s \geq 0$, $i \geq 1$. This provides the following identity:

$$
S_{1}+S_{2}=\sum_{s=0}^{\infty} \sum_{i=1}^{\infty} \delta_{s, k} \delta_{i, l} \frac{x^{s}}{s !} y^{s+i} \frac{1}{i}=\frac{x^{k}}{k !} \frac{y^{k+l}}{l},
$$


where $S_{1}$ and $S_{2}$ are given by

$$
\begin{gathered}
S_{1} \equiv \alpha \sum_{s=0}^{\infty} \sum_{i=1}^{\infty} s\left[b_{k, l}(s-1, i+1)-b_{k, l}(s, i)\right] \frac{x^{s}}{s !} \frac{y^{s+i}}{s+i} \\
=\alpha\left[x G_{k, l}(x, y)-M_{k, l}(x y)\right]-\alpha x \frac{\partial}{\partial x} G_{k, l}(x, y), \\
S_{2} \equiv \mu \sum_{s=0}^{\infty} \sum_{i=1}^{\infty}\left[b_{k, l}(s, i-1)-b_{k, l}(s, i)\right] \frac{x^{s}}{s !} y^{s+i}=\mu y^{2} \frac{\partial}{\partial y} G_{k, l}(x, y)-\mu y \frac{\partial}{\partial y} G_{k, l}(x, y),
\end{gathered}
$$

after some calculations. Thus, (4.16) can be written as

$$
-\alpha x \frac{\partial}{\partial x} G_{k, l}(x, y)+\mu y(y-1) \frac{\partial}{\partial y} G_{k, l}(x, y)=\frac{x^{k}}{k !} \frac{y^{k+l}}{l}+\alpha M_{k, l}(x y)-\alpha x G_{k, l}(x, y),
$$

i.e. a partial differential equation for $G_{k, l}(x, y)$ provided the function $M_{k, l}(x y)$ is known.

We start by deriving two first integrals to (4.17). The associated characteristic system is

$$
\frac{\mathrm{d} x}{-\alpha x}=\frac{\mathrm{d} y}{-\mu y(1-y)}=\frac{\mathrm{d} G_{k, l}(x, y)}{\left(x^{k} / k !\right)\left(y^{k+l} / l\right)+\alpha M_{k, l}(x y)-\alpha x G_{k, l}(x, y)} .
$$

The first equality in (4.18) yields the integral $x=c_{1}[y /(1-y)]^{\alpha / \mu}, c_{1}$ being an arbitrary constant. The second equality leads to the linear differential equation

$$
\frac{\mathrm{d} G_{k, l}(x, y)}{\mathrm{d} y}+\frac{\alpha x G_{k, l}(x, y)}{-\mu y(1-y)}=\frac{\left(x^{k} / k !\right)\left(y^{k+l} / l\right)+\alpha M_{k, l}(x y)}{-\mu y(1-y)} .
$$

Its solution is found to be

$$
G_{k, l}(x, y)=b(y) \exp \left[c_{1}\left(\frac{y}{1-y}\right)^{\alpha / \mu}\right],
$$

where $b(y)$ can be determined by the variation of constants methods. Then

$$
\begin{aligned}
b(y)=c_{2}+\int_{0}^{y} & \exp \left[-c_{1}\left(\frac{w}{1-w}\right)^{\alpha / \mu}\right] \\
& \times \frac{\left(c_{1}^{k} / k !\right)[w /(1-w)]^{\alpha k / \mu}\left(w^{k+l} / l\right)+\alpha M_{k, l}\left(c_{1} w[w /(1-w)]^{\alpha / \mu}\right)}{-\mu w(1-w)} \mathrm{d} w,
\end{aligned}
$$

with an arbitrary constant $c_{2}$.

We can now derive the general solution to (4.17). Let us write $c_{2}=f\left(c_{1}\right)$ for some function $f$ to be determined. Making the change of variable $v=(w / y)(1-y) /(1-w)(\in(0,1))$, we obtain, from (4.19), (4.20), and with $c_{1}$ given above,

$$
\begin{aligned}
G_{k, l}(x, y)= & \mathrm{e}^{x} f\left[x\left(\frac{y}{1-y}\right)^{-\alpha / \mu}\right] \\
& -\mathrm{e}^{x} \frac{1}{\mu} \frac{x^{k}}{k !} \frac{y^{k+l}}{l} \int_{0}^{1} v^{k+l-1+k \alpha / \mu}\left[\frac{1}{1+y(v-1)}\right]^{k+l} \exp \left(-x v^{\alpha / \mu}\right) \mathrm{d} v \\
& -\mathrm{e}^{x} \frac{\alpha}{\mu} \int_{0}^{1} v^{-1} M_{k, l}\left(x y \frac{v^{1+\alpha / \mu}}{1+y(v-1)}\right) \exp \left(-x v^{\alpha / \mu}\right) \mathrm{d} v
\end{aligned}
$$


Let us show that $f$ is identically null. First, it can be seen from (4.21) that the function $f\left(x[y /(1-y)]^{-\alpha / \mu}\right)$ can be expanded near the point $(0,0)$ as $\sum_{r=0}^{\infty} x^{r} h_{r}(y)$, the functions $h_{r}$ being analytic near 0 . By differentiation, this implies that

$$
\left(\frac{y}{1-y}\right)^{-\alpha j / \mu} f^{(j)}(0)=j ! h_{j}(y), \quad j \geq 0 .
$$

As $[y /(1-y)]^{-\alpha j / \mu}$ has an essential singularity at 0 when $j>0$, this is possible only if $f^{(j)}(0)=0$ for all $j>0$. Thus, the function $f$ is identically constant. From (4.21) with $y=0$, this constant is 0 since $G_{k, l}(x, 0)=0=M_{k, l}(x, 0)$ by definition.

To obtain the $b_{k, l}(s, i)$, we determine the coefficients of $x^{s} y^{s+i} / s !(s+i)$ in the right-hand side of (4.21) (where $f \equiv 0$ ). So, we substitute (4.15) for $M_{k, l}$ and we expand the two other functions of $x$ and $y$ as power series. In the first integral, the sought coefficient is given by

$$
-\frac{1}{\mu(k !) l}\left(\begin{array}{c}
-k-l \\
s+i-k-l
\end{array}\right) \frac{1}{(s-k) !} \int_{0}^{1} v^{k+l-1+k \alpha / \mu}(\nu-1)^{s+i-k-l}\left(1-v^{\alpha / \mu}\right)^{s-k} \mathrm{~d} \nu .
$$

Expressing $\left(\begin{array}{c}-k-l \\ s+i-k-l\end{array}\right)$ as $[(k+l) /(l+i)]\left(\begin{array}{c}s+i \\ k+l\end{array}\right)(-1)^{s+i-k-l}$, it can be written as $(1 / l) \phi_{k, l}(s, i)$ in the notation of (4.12). The coefficient in the second integral is derived in the same way, hence (4.13) for $b_{k, l}(s, i)$. Note that the sum in (4.13) disappears if $k+l>s$. Otherwise, the $b_{k, l}(j-1,1)$ are obtained from (4.13) with $i=0$ and $b_{k, l}(s, 0)=0$, which yields (4.14).

\section{Illustrations in insurance}

Compartment Markovian models provide a useful tool for the actuarial modelling of health insurance plans. See, e.g. Haberman and Pitacco (1999) and Denuit and Robert (2007). For the epidemic risk in a company, the three compartments of SIR models play significantly different roles. Premiums are perceived from the group of susceptible policyholders and cover the healthcare benefits paid to infected insureds. This is the approach developed by Feng and Garrido (2011) using a deterministic SIR model.

In the present Markovian context, the insurer reimburses part of the medical expenses to each infective continuously at a rate $c_{1}$ per time unit, and pays also a lump sum of amount $c_{2}$ immediately at the moment of its removal. Thus, on the insurance liability side, the expected value of the payments is given by $\mathbb{E}\left[\int_{0}^{T} c_{1} I_{u} \mathrm{~d} u+c_{2} R_{T}\right]$. The susceptibles have to pay premiums continuously at a rate $\pi$ per time unit as long as they remain healthy. Thus, on the revenue side, the expected value of all the received premiums is equal to $\mathbb{E}\left[\int_{0}^{T} \pi S_{u} \mathrm{~d} u\right]$. Now, by the classical equivalence principle (1.1), the premium level $\pi$ is chosen in such a way that

$$
\pi=\frac{c_{1} \mathbb{E}\left(A_{T}\right)+c_{2}\left[N-\mathbb{E}\left(S_{T}\right)\right]}{\mathbb{E}\left(B_{T}\right)} .
$$

Equation (5.1) requires the calculation of $\mathbb{E}\left(S_{T}\right), \mathbb{E}\left(A_{T}\right)$, and $\mathbb{E}\left(B_{T}\right)$. This can be done using the results of the previous sections, as illustrated below.

General and fatal epidemics. We consider these two models when there are initially $n=30$ susceptibles and $m=3$ infectives $(N=33)$. The removal rate is $\mu=1$, while the infection rates, $\beta=\alpha / N$ and $\beta_{r}=\alpha /(N-r)$, respectively, are viewed as a function of $\alpha$.

Equation (2.8) allows us to determine the probability mass function of $S_{T}$ for both models. In Figure 1 we show the graphs obtained when $\alpha=1$ and $\alpha=1.5$. The final susceptible size has a tendency to be less important when $\alpha=1.5$ and for the fatal epidemic, which is natural 

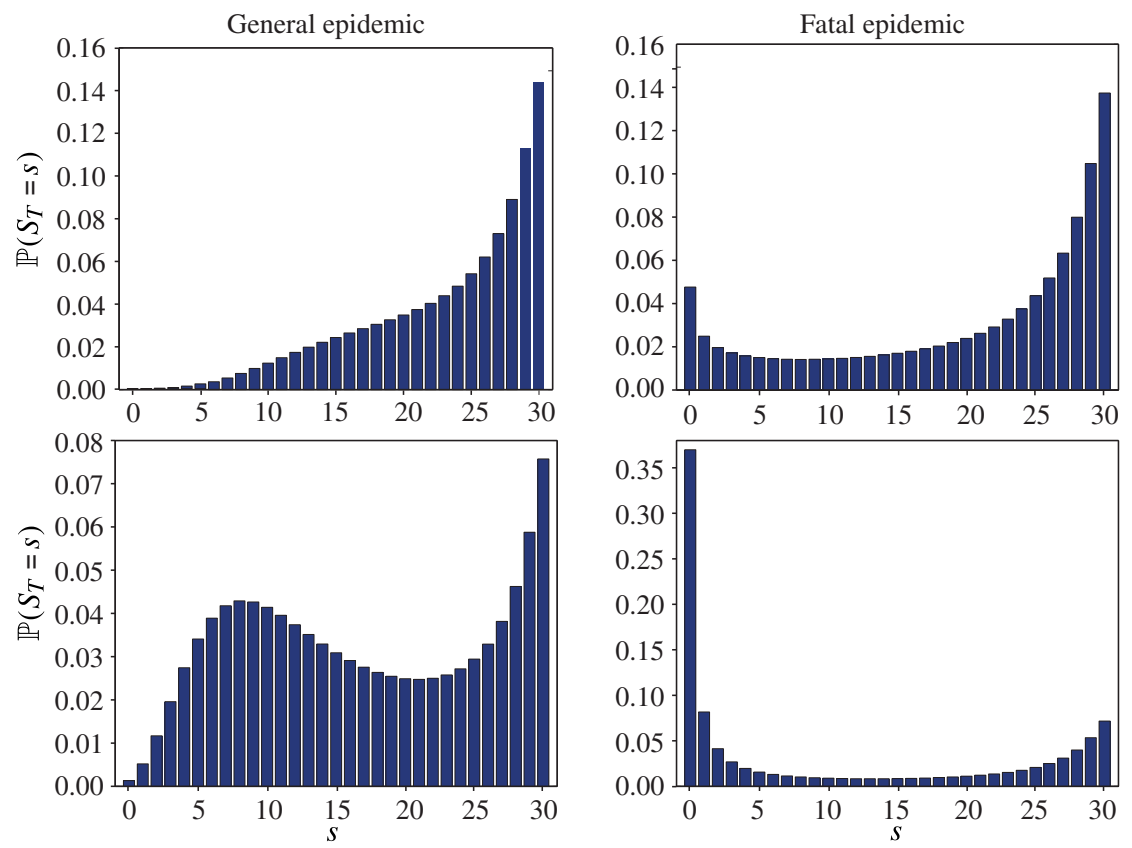

FIGURE 1: Probability mass function of $S_{T}$ when $n=30, m=3, \mu=1$ and $\alpha=1$ (upper) or 1.5 (lower).
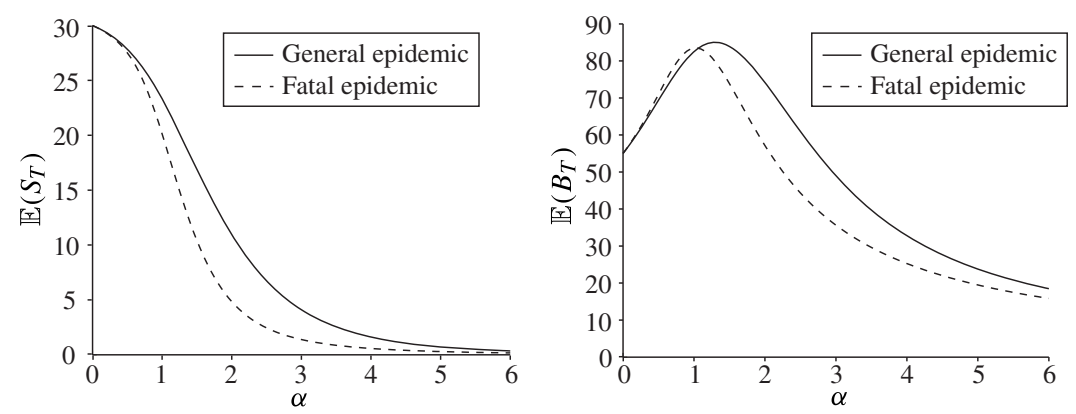

FIGURE 2: Plots of $\mathbb{E}\left(S_{T}\right)$ and $\mathbb{E}\left(B_{T}\right)$ when $n=30, m=3, \mu=1$, and $\alpha$ varying from 0 to 6 .

since the infection rate is larger. In Figure 2 we have the graph of $\mathbb{E}\left(S_{T}\right)$ and $\mathbb{E}\left(B_{T}\right)$ when $\alpha$ varies from 0 to 6 . They are obtained from (2.8) and (3.10), (4.3), (4.4), (4.13), and (4.14). We recall that $\mathbb{E}\left(A_{T}\right)$ is simply given by (2.11). These expectations are rather different between the two models when $\alpha$ takes intermediate values (between 1 and 5). When $\alpha$ is large (small), the infection is so strong (weak) that the general and fatal epidemics yield similar results. The function $\mathbb{E}\left(B_{T}\right)$ is unimodal, which can be explained intuitively: when $\alpha$ is small, $\mathbb{E}\left(B_{T}\right)$ increases with $\alpha$ because the duration of the epidemic increases too, but when $\alpha$ is large, $\mathbb{E}\left(B_{T}\right)$ decreases to 0 because the epidemic becomes too severe. Note that $\mathbb{E}\left(S_{T}\right)$ tends to 0 much faster.

Next, we examine the above insurance contract under the same conditions and when the epidemic costs are $c_{1}=1$ and $c_{2}=2$. The graph of the premium $\pi$ evaluated by (5.1) is shown in Figure 3. As expected, for both models, the bigger the infection parameter $\alpha$, the higher the premium level $\pi$. 


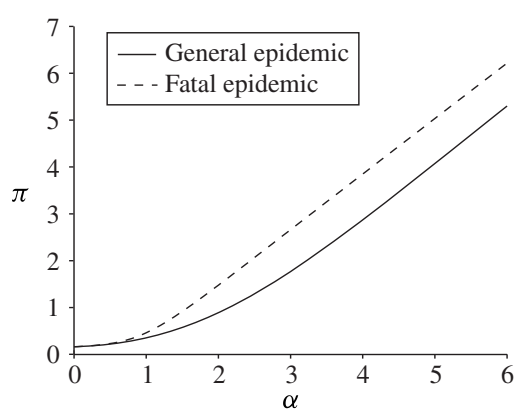

FIGURE 3: Premium $\pi$ when $c_{1}=1, c_{2}=2$ and $n=30, m=3, \mu=1$, for $\alpha$ varying from 0 to 6 .

Exponentially-dependent rates. We now consider an SIR epidemic in which the removal and infection rates are exponential functions of $r$ of the form

$$
\mu_{r}=\mu(1+r)^{u} \quad \text { and } \quad \beta_{r}=\frac{\alpha}{N}(1+r)^{v},
$$

where $u$ and $v$ can be viewed as powers of removal, positive or not. For $u=v=0$, the model becomes the general epidemic. The removal rate $\mu_{r}$ increases (decreases) with the number of removals when $u>0(u<0)$; the same arises for the infection rate $\beta_{r}$ in the function of $v$. Our goal is to show the influence of the parameters $u$ and $v$. The other parameters in the rates are $\mu=1$ and $\alpha=2$, and the initial population contains $n=30$ susceptibles and $m=3$ infectives.

For $S_{T}$, we see from (2.8) that its distribution depends only on the difference of powers $u-v$. In Figure 4 we show the graphs obtained when $u-v=-1,0,1,2$. The final susceptible size has a tendency to be more important for larger values of $u-v$ since the difference between the removal and infection rates is then larger.

For $A_{T}$ and $B_{T}$, their distributions depend on $u$ and $v$ taken separately. The insurance contract is proposed to cover again the costs $c_{1}=1$ and $c_{2}=2$. The graphs of $\mathbb{E}\left(B_{T}\right)$ and $\pi$ are shown in Figure 5 when $u-v=-1,0$, and 1 and $u$ varies from -1 to 2 . They are obtained from (3.10), (3.15) and (5.1), (2.8), and (2.10). We see that $\mathbb{E}\left(B_{T}\right)$ varies when $u-v$ is fixed, sometimes strongly. Note also that $\pi$ can be decreasing when $u-v<0$ and $u<0$. In Figure 6 we show the graphs of $\mathbb{E}\left(B_{T}\right)$ and $\pi$ when $u=-0.5,0$ or 1 and $v$ varies from -2 to 2 . The function $\mathbb{E}\left(B_{T}\right)$ is unimodal here too. It is bigger for $u$ and $v<0$, and it tends to 0 when $v$ is large enough since the susceptibles are then infected very quickly. The function $\pi$ is increasing, with a nonmonotonous speed.

Single change in infection. To close, we consider a modified general epidemic for which the value of the infection rate changes when the number of removals exceeds a given critical value, i.e.

$$
\beta_{r}= \begin{cases}\frac{\alpha_{1}}{N} & \text { if } r \leq r^{*}, \\ \frac{\alpha_{2}}{N} & \text { if } r>r^{*},\end{cases}
$$

where $\alpha_{1}, \alpha_{2}$ are two constants. When $r^{*}=N$, the model reduces to the general epidemic of rate $\alpha_{1}$. A change in the infection rate could arise when the health services throw an alert further to the recording of a large number of removed cases. The authorities expect the population to change a little in its behaviour to better protect it from the infection, yielding $\alpha_{2} \leq \alpha_{1}$. A similar situation could also exist with household epidemic models as the behaviour within a household 

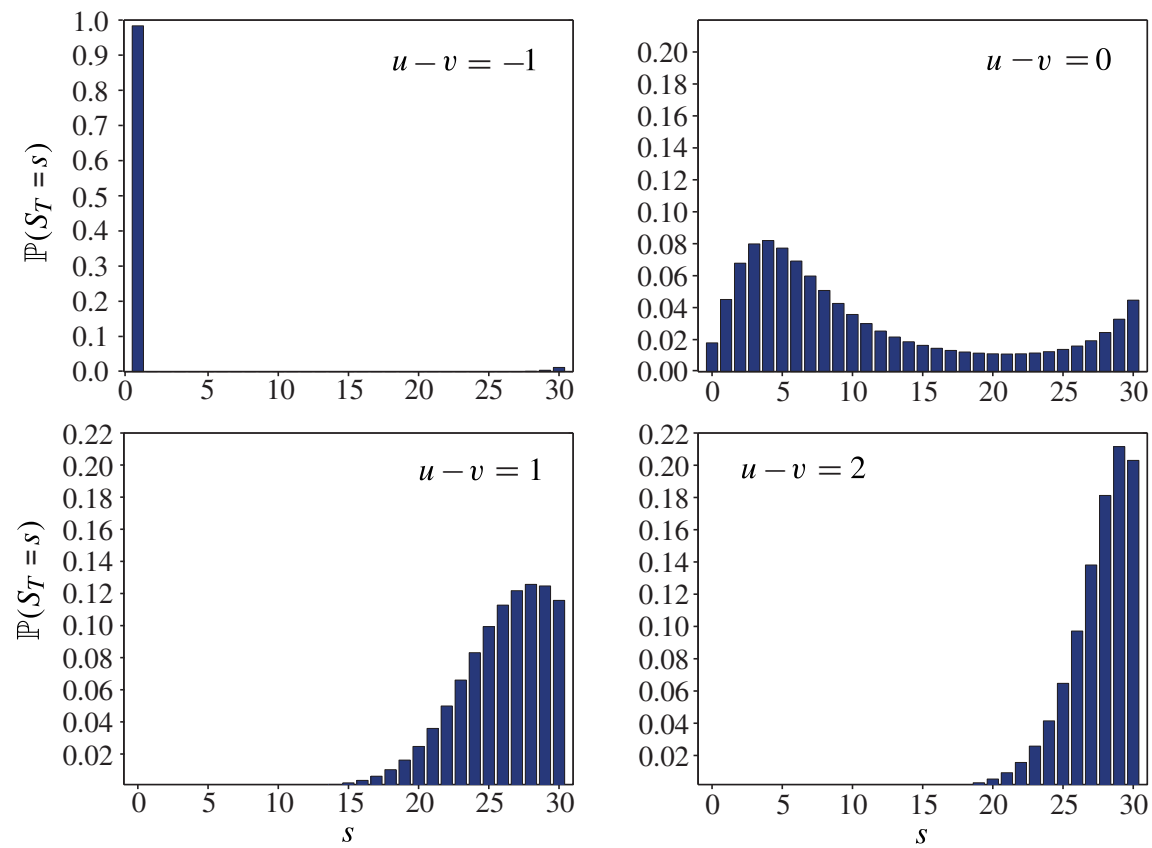

FIGURE 4: Probability mass function of $S_{T}$ when $n=30, m=3, \mu=1, \alpha=2$ and $u-v=-1,0,1,2$.
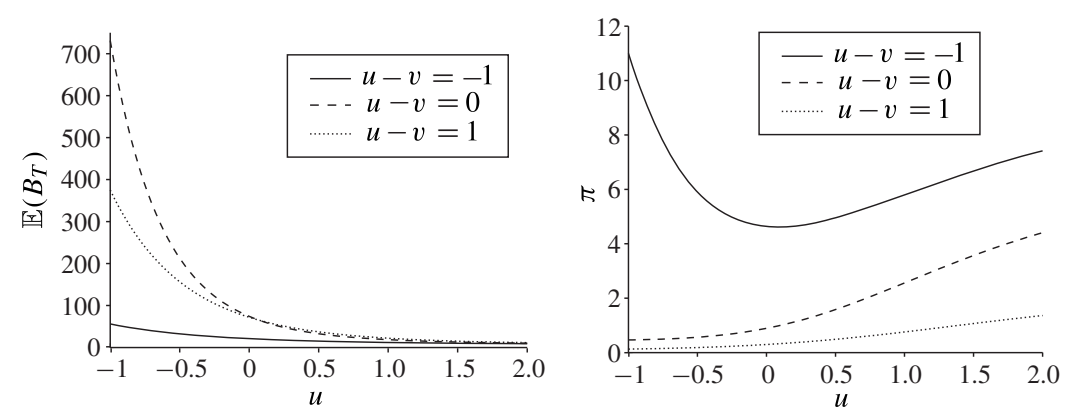

Figure 5: Plots of $\mathbb{E}\left(B_{T}\right)$ and $\pi$ when $c_{1}=1, c_{2}=2$ and $n=30, m=3, \mu=1, \alpha=2, u-v=-1,0,1$ and $u$ varying from -1 to 2 .

is expected to change once a case has been detected. For works related to this point, see, e.g. Ball et al. (2007) and Ball et al. (2008).

For instance, let us fix $\alpha_{1}=2$ and let $\alpha_{2}$ vary from 0 to $\alpha_{1}$, for three different values of $r^{*}$. The population has initially $n=30$ susceptibles and $m=3$ infectives, and the removal rate is $\mu=1$ (independent of $r$ ). The cost components are $c_{1}=1$ and $c_{2}=2$.

In Figure 7 we show the graphs of $\mathbb{E}\left(S_{T}\right), \mathbb{E}\left(A_{T}\right), \mathbb{E}\left(B_{T}\right)$, and $\pi$ as a function of $\alpha_{2}$, for $r^{*}=5,10$ or 20. For $\alpha_{2}=2$, these quantities correspond to those obtained for the general epidemic. The observed monotonicity of $\mathbb{E}\left(S_{T}\right)$ and $\mathbb{E}\left(A_{T}\right)$, and $\pi$ is intuitive. In particular, the premium level increases with the infection rate and the threshold level $r^{*}$. For $\mathbb{E}\left(B_{T}\right)$, its value for $\alpha_{2}=0$ is larger than for the general epidemic, as expected. Actually, the function $\mathbb{E}\left(B_{T}\right)$ presents a peak which becomes less marked when $r^{*}$ is large. 

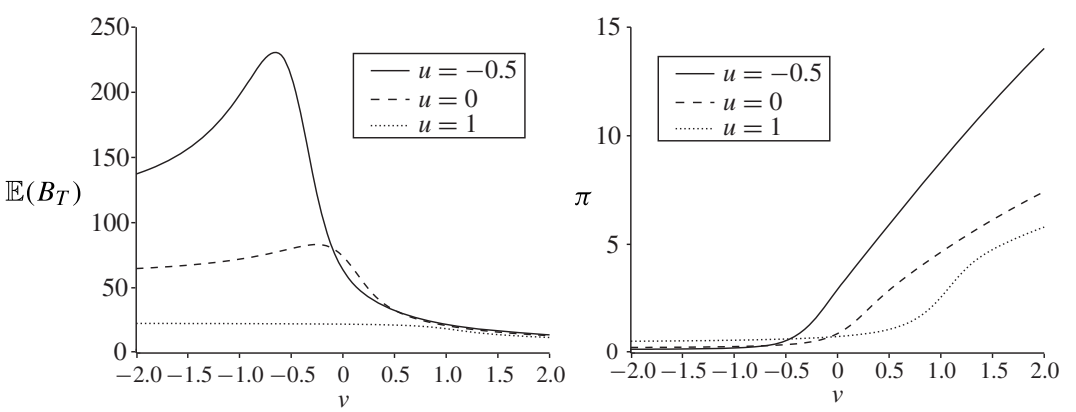

Figure 6: Plots of $\mathbb{E}\left(B_{T}\right)$ and $\pi$ when $c_{1}=1, c_{2}=2$ and $n=30, m=3, \mu=1, \alpha=2, u=-0.5,0,1$ and $v$ varying from -2 to 2 .
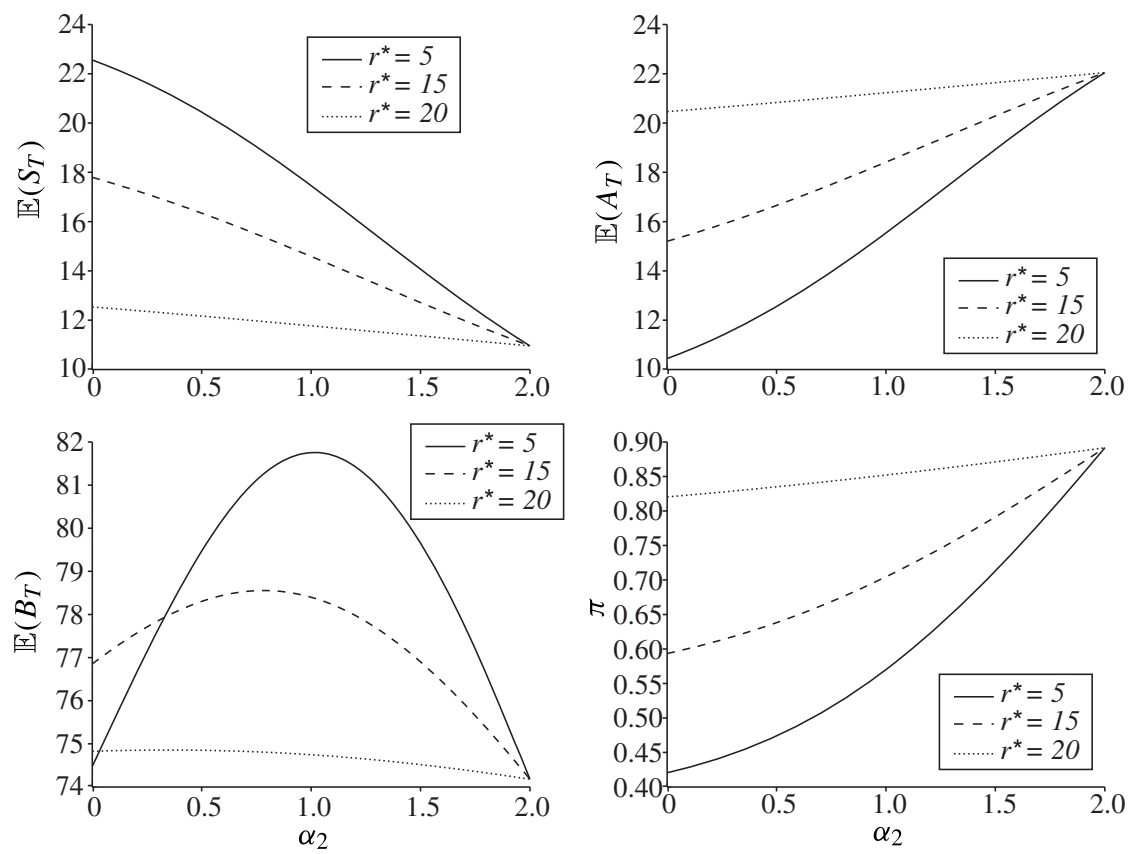

Figure 7: Plots of $\mathbb{E}\left(S_{T}\right), \mathbb{E}\left(A_{T}\right), \mathbb{E}\left(B_{T}\right)$ and $\pi$ when $c_{1}=1, c_{2}=2$ and $n=30, m=3, \mu=1, r^{*}=$ $5,10,20, \alpha_{1}=2$ and $\alpha_{2}$ varying from 0 to 2 .

\section{Conclusions}

The main contribution of this paper is two-fold. On one hand, we considered a generalized SIR model that allows the removal and infection rates to depend on the number of removal cases. On the other hand, we proposed a simple actuarial approach to ensure that the financial risk of an epidemic in a company is covered. This led us to focus on the expected infectivity and susceptibility times which allow for the setting of insurance premiums based on the classical equivalence principle. 


\section{Acknowledgements}

We are grateful to both anonymous referees for very helpful and constructive comments. C. Lefèvre received support from the ANR project LoLitA of the French Agence Nationale de la Recherche. The research of M. Simon was supported by the Belgian FNRS through an FRIA research grant.

\section{References}

Andersson, H. And Britton, T. (2000). Stochastic Epidemic Models and Their Statistical Analysis (Lecture Notes Statist. 151). Springer, New York.

Ball, F. AND O'NeILl, P. D. (1993). A modification of the general stochastic epidemic motivated by AIDS modelling. Adv. Appl. Prob. 25, 39-62.

Ball, F., O’NeILl, P. D. AND PIKe, J. (2007). Stochastic epidemic models in structured populations featuring dynamic vaccination and isolation. J. Appl. Prob. 44, 571-585.

BALL, F. G. (1986). A unified approach to the distribution of total size and total area under the trajectory of infectives in epidemic models. Adv. Appl. Prob. 18, 289-310.

Ball, F. G., KNock, E. S. AND O’Neill, P. D. (2008). Control of emerging infectious diseases using responsive imperfect vaccination and isolation. Math. Biosci. 216, 100-113.

Chen, H. And Cox, S. H. (2009). An option-based operational risk management model for pandemics. N. Amer. Actuarial J. 13, 54-76.

Daley, D. and Gani, J. (1999). Epidemic Modelling. Cambridge University Press.

Denuit, M. And Robert, C. (2007). Actuariat des Assurances de Personnes. Economica, Paris.

FENG, R. AND Garrido, J. (2011). Actuarial applications of epidemiological models. N. Amer. Actuarial J. 15, $112-136$.

Gani, J. And Jerwood, D. (1972). The cost of a general stochastic epidemic. J. Appl. Prob. 9, 257-269.

Gleissner, W. (1988). The spread of epidemics. Appl. Math. Comput. 27, 167-171.

Haberman, S. and Pitacco, E. (1999). Actuarial Models for Disability Insurance. Chapman and Hall, Boca Raton.

O’Neill, P. D. (1997). An epidemic model with removal-dependent infection rate. Ann. Appl. Prob. 7, 90-109.

PiCARD, P. (1980). Applications of martingale theory to some epidemic models. J. Appl. Prob. 17, 583-599.

PiCARD, P. ANd LefÈvre, C. (1990). A unified analysis of the final size and severity distribution in collective Reed-Frost epidemic processes. Adv. Appl. Prob. 22, 269-294.

Picard, P. And Lefèvre, C. (1993). Distribution of the final state and severity of epidemics with fatal risk. Stoch. Process. Appl. 48, 277-294. 